Talitha da Cunha Pires Middleton

\title{
Declínio de aves no Arco do Desmatamento Amazônico
}

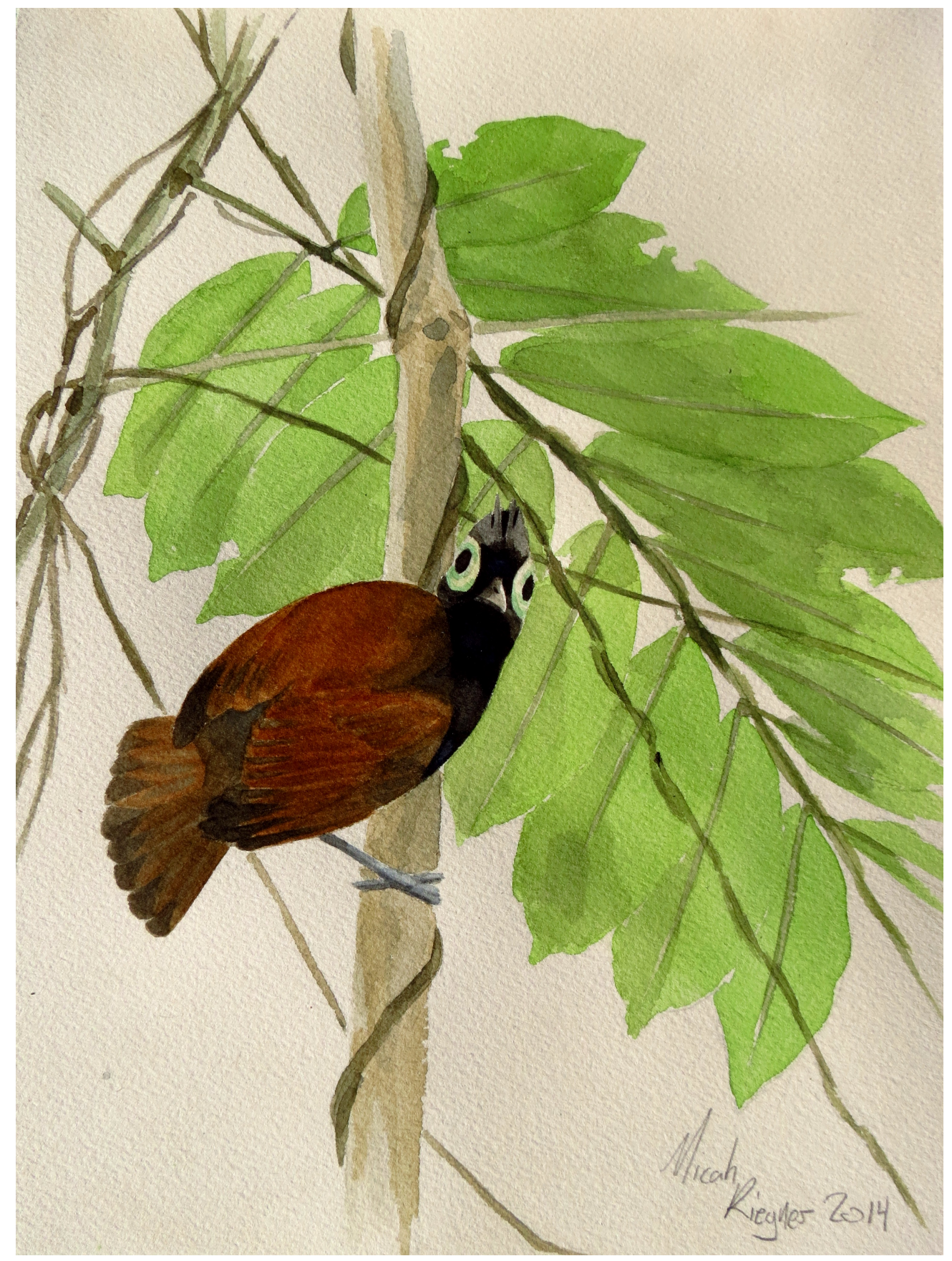

São Paulo

2016 


\section{Talitha da Cunha Pires Middleton}

\section{Declínio de aves no Arco do Desmatamento Amazônico}

Tese apresentada ao Instituto de Biocências da Universidade de São Paulo, para a obtenção de Título de Doutor em Ciências Biológicas, na Área de Zoologia.

Orientadora: Dra. Elizabeth Höfling

Coorientador: Dr. Carlos Augusto Peres

São Paulo

2016 


\section{Ficha Catalográfica}

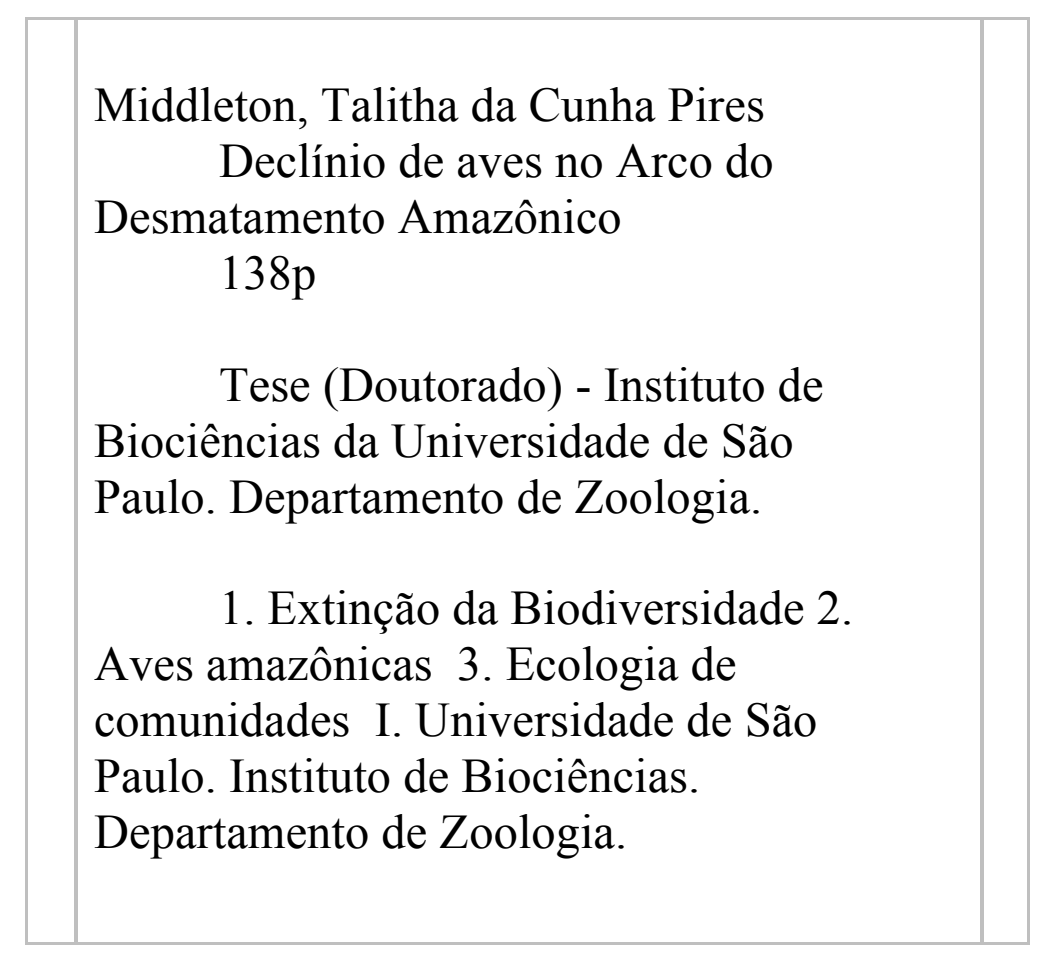

\section{Comissão Julgadora}

Prof(a). Dr(a).

Prof(a). Dr(a).
Prof(a). Dr(a).

Prof(a). Dr(a).

$\overline{\text { Profa. Dra. Elizabeth Höfling }}$

Presidente 


\section{DEDICATÓRIA}

Ao George, com amor 


\section{EPÍGRAFE}

"Art is a lie that makes us realize truth"

Pablo Picasso 


\section{AGRADECIMENTOS}

A minha orientadora Profa. Dra. Elizabeth Höfling, por assumir a responsabilidade de me orientar em uma área diferente, confiar que eu poderia fazer este projeto e por me dar tanta liberdade para executá-lo, me apoiando em momentos decisivos. Parabéns pela sólida carreira!

Ao meu coorientador Prof. Dr. Carlos Peres por me apresentar a Amazônia, dividir conhecimentos sobre esse bioma, além de me receber na Universidade de East Anglia, no Reino Unido, para o período sanduíche e por sua constante ambição e coorientação.

Aos financiadores desta pesquisa, pois sem eles nada disso teria acontecido: Coordenação de Aperfeiçoamento de Pessoal de Nível Superior (CAPES) pela bolsa de doutorado; Programa de Doutorado Sanduíche no Exterior (PDSE-CAPES) pela bolsa de doutorado sanduíche; Programa de Excelência Acadêmica (PROEX) pelo auxílio financeiro para a realização das campanhas de campo e Prof. Dr. Marcelo Carvalho, coordenador do Programa em Zoologia da Pós-Graduação do Instituto de Biociências, pelo apoio; Rufford Small Grant Foundation, pelo financiamento de boa parte da campanha de campo e Idea Wild pelo material.

Ao Hotel Cristalino Lodge por me dar todo apoio necessário e hospedagem no período em campo no Cristalino. À dona Vitória e Alexandre por seus comprometimentos com a conservação, apoio e amizade durante a execução do projeto. Aos guias do Cristalino, Francisco e Jorge.

A Andy Siemens (BirdLife International-UK), Susan Evans (UCL-UK) e Mônica Toledo Piza-Ragazzo (IB-USP) pelas cartas de recomendação para que eu conseguisse os financiamentos. Assim como a tradução das muitas solicitações, por George, Micky e Alice.

Em especial, durante o doutorado, à Profa. Dra. Mônica T. Piza-Ragazzo, pelo apoio constante à minha formação, amizade e o Prof. Dr. Clodoaldo Ragazzo por me recomendar ao Prof. Dr. Gilberto de Paula que me ajudou com os modelos.

Ao Prof. Dr. Jean-Paul Metzger por abrir a porta de seu laboratório no início do doutorado, dedicar seu tempo para ler minha qualificação, tirar minhas dúvidas de campo, enfim, por seu carinho e apoio constantes.

Ao Prof. Dr. Milton Ribeiro, por sua alegria, por me receber na Unesp-Rio Claro, quando comecei mapear as imagens Landsat e ao seu irmão John pelos cálculos de parte das métricas de paisagem. A Felipe e Talita pelo apoio naquela semana. 
À Profa. Dra. Renata Pardini, por me educar como bióloga e me apoiar sempre. Ao Prof. Dr. Fernando Portela Marques, por sua amizade, apoio e momentos de descontração. Ao Prof. Dr. Paulo Inácio Prado por sua calma em tirar dúvidas e por me apresentar o programa R! Ao Prof. Dr. Rui Murieta, por sua amizade e conhecimentos. Ao Prof. Dr. Sergio Matioli pelo apoio em toda minha carreira. Ao Prof. Dr. Walter Neves por suas aulas maravilhosas e perguntas perspicazes. Aos demais professores da Zoologia e Ecologia por todo conhecimento transmitido.

Durante o doutorado, pelo apoio logístico e alegria das meninas da secretaria de Pós-graduação do IB, Lilian e Érika, o pessoal da biblioteca, os porteiros, secretários, funcionários e professores.

A todos da Universidade de São Paulo-IB, por possibilitarem minha formação como bióloga. Foram muitos anos nesses prédios, cheios de lindas teias de aranha...

Aos que merecem agradecimentos com letras garrafais: Mario Pacheco, Marito, por todos o seu apoio com a parte estatística e por seu calmo "tá bom"! A Alexander Lees pelo auxílio nas identificações das vocalizações, pela coleta dos dados de 2004/06 e pela leitura do primeiro capítulo. Ao Tank, por seus conselhos, críticas, amizade e leitura do segundo capítulo.

Nas campanhas de campo, agradeço a todos que me acompanharam, por todas as madrugadas, pelos esforços além do limite, por todos os carrapatos pegos, por todas as aventuras, perrengues, alegrias, registros e experiências e histórias compartilhadas na Amazônia. E são muitas! Luísa, Michelle, Ben, Kamilla, Tom, foi muito bom tê-los como estagiários em campo! Vocês foram muito guerreiros! Fico muito feliz em saber que o projeto foi uma inspiração para muitos que seguirão estudando aves, mesmo tendo experimentado a dor de madrugar.... Em especial, ao João Carlos (JC), pois sua companhia foi essencial para a realização das campanhas de campo, desde o perfeccionismo para abrir as picadas, por madrugar pontualmente, pelo constante apoio com as redes, por seus conhecimentos da natureza, mesmo com as várias vezes dizendo que iria desistir, você foi ficando....Graças! A Alice (Pequititica) por ter me apoiado nos meses mais difíceis, no meio do campo quando o fim estava longe, mas o cansaço dos dois primeiros meses já doíam nos ossos. Sua amizade e companheirismo foi um presente de doutorado e sem você e seu chá (txai) que nos mantinha o dia todo, tudo seria impossível. Quantas histórias compartilhadas para relembrar! Também agradeço por me receber em sua casa Norwich para o período sanduíche e por me apresentar aos queridos housemates Thomas, Rachel, Maud, Leticia e Tiger! Ao Odair por sua alegria e companheirismo em campo e pelo último dia que sem ele eu não teria conseguido finalizar da forma como foi. Ao René por me acompanhar no último mês, me aliviando tanto, dirigindo o carro nas estradas mais assombrosas que conheci (com buracos do tamanho de um carro), por me fazer rir e aliviar meu cansaço quando já me restava pouca força. Ao Micah Reigner por ter nos acompanhado no Cristalino e em um fragmento e por compartilhar de seu entusiasmo com todas as novas descobertas e por sua pintura que ilustra a página inicial da tese, além de duas filmagens que estão no 
vídeo entregue aos proprietários de Alta Floresta. Pelo filme com produção de AdrianoEspinola.

Acesso:http://www.rufford.org/projects/talitha_cunha_pires_middleton.

A seu Geraldo por ter me levado, de moto, para reconhecer muitas das áreas. Por seu conhecimento sobre a natureza e seu filho Alex e esposa Nélia. A seu Zé Piva (e seu filho Leandro) por compartilhar seu profundo conhecimento sobre plantas e animais e por estar sempre disposto a me ajudar ou pegar um coco ou castanha-do-Pará...

Especialmente, aos proprietários de Alta Floresta e Carlinda por permitirem a realização dos trabalhos de campo e por nos receber sempre com uma boa conversa, bolo, café e alguns almoços. As histórias de vocês deveriam estar em um livro! Quantas transformações vocês viram....Aos muitos moradores de Alta Floresta que facilitaram minha vida em vários sentidos, desde alugando carro, os meninos no posto de gasolina, do restaurante, ao moço da loja de pescaria, ao médico! Aos professores da UNEMAT, Darwin (Bocó) e em especial ao professores Mendelson e Maga por todo o carinho e apoio, além de nos receber em sua ilha paradisíaca. Vocês são demais! À Vani pelo aluguel da casa que tornou possível que eu e mais duas cientistas, Babi e Ana, trabalhássemos na região, pois em tempos de hidrelétricas torna-se difícil achar uma casa para alugar, ainda mais uma que incluísse os dois salsichas mais lindos Zeca e Bob... A Felipe e Mariana por me receberem em sua casa na segunda campanha e nos deixar tão à vontade.

Ao Prof. Dr. Kevin Johnson, da Universidade de Illinois, por me aceitar no doutorado que acabei não podendo fazer. Sou muito grata! Enquanto morei na Inglaterra, ao Nigel Colar e Andy Siemens pelo estágio voluntário na BirdLife International e apoio. A Susan Evans (UCL) pelo apoio constante e estágio quando estava na Inglaterra e por seu conhecimento profundo sobre os fósseis.

Aos colegas recentes de laboratório: Pauline (e Romain), obrigada por sua companhia, amizade e suporte, Daniel e Graziela. Ao colegas de disciplina de pósgraduação por compartilharem conhecimentos: Erika, Vitor, Fábio, Marina, Melinas, Greet, Thayná, Jayme, Leandro, por seus conselhos e apoio. Paula, pelas imagens Landsat georrerferenciadas e apoio. Na Universidade de East Anglia, pelos momentos de alegria e muito empenho, a Gabi, Maíra, Vanessa, Rodrigo, Vivi, Tiago, Davi, JB, Maria, Anderson, Mark, Jamile, Maxin, Babi, Daniela e Marta à Profa. Dra Aldina. A Alina, Paul e Tom, pelo auxílio com os isótopos.

Aos meus amigos queridos, que mesmo com tantas ausências nunca desistiram de mim, de se comunicar: os de todo dia, os das antigas, os da faculdade, da Inglaterra, aos facilitadores da minha vida Guilhermina, Matilde e Eliel. E aos guerreiros que me inspiram diariamente: Camila, Letícia, Maíra, Renato Maranhão e a pequena Hazel por toda a superação e amizade! 
Pelo apoio constante da minha família, sempre! Mãe, sou eternamente grata a tudo que fez por nós seus filhos, com tanta leveza, alegria e sabedoria. Você é nossa inspiração! Pai e Kuka, obrigada por todo apoio e entusiasmo de sempre! Fefe, meu irmão, obrigada por me ensinar tanto e com tanto amor! As minhas primeiras grandes amigas, irmãs amadas, Tathi e Tamy e a meus queridos cunhados Caio e Rodrigo. A meus avós e minha vozinha querida...em referência ao seu programa favorito...que saudade de você! To my adorable new family in the Middletons, Richard, Diana, Lucy, Angus, Jimbo and Emma, for giving me open arms, making me part of the family and being marvellous and fun people! I have learnt a lot from your perceptive questions and by your interest in all subjects. And, especially, for sharing your love of nature and being so open to my culture and country. Em especial aos meus sobrinhos, Archie, Isabelle, Miguel, Jemima, Barney, Herbie e dois que nascerão este ano, por sempre me colocarem um sorriso no rosto.

Por último, mas primeiro, gostaria de agradecer ao George! Por entender que alguns de nossos sonhos seriam prorrogados, que a distância seria uma realidade por alguns, muitos, meses e que o dia a dia teria uma rotina sacrificante. Com tudo isso, teve amor, paciência, frustrou-se, animou-se com os resultados, vibrou com as pequenas conquistas, quase desistiu do doutorado, mas voltou revigorado, empenhou-se para traduzir os muitos pedidos de financiamento para o campo, fez trabalho braçal, digitou dados, passou bastante tempo na frente do computador inventando tarefas, como ele falava "administrativas" só para me acompanhar. Quando planejava as férias para me ajudar em campo, aliviava minhas responsabilidades; era motorista, cozinheiro, abria picada, coordenava a equipe, me cuidou, alimentou, pediu que fosse dormir e cuidou de nossa casa para meu regresso. Respeitou minha decisão profissional, meu amor à pesquisa e conservação das aves e, acima de tudo, compartilhou dessa paixão! Obrigada por ser meu companheiro na vida e me trazer tanta alegria! Que sortuda que eu sou..

À Amazônia, por me presentear com seus sons, cheiros e diversidade... 


\section{ÍNDICE}

INTRODUÇÃO GERAL

CAPÍTULO 1: A rápida extinção local dos papa-formigas no Arco do Desmatamento Amazônico

Resumo

1. Introdução

2. Materiais e Métodos

16

3. Resultados

4. Discussão

5. Considerações Finais

6. Referências bibliográficas

7. Anexos

CAPÍTULO 2: Efeitos sinergéticos entre fragmentação e degradação de habitat aceleram as extinções locais de aves de sub-bosque no Arco do Desmatamento Amazônico

Resumo

1. Introdução

57

2. Materiais e Métodos

3. Resultados

4. Discussão

80

5. Considerações Finais

90

6. Referências bibliográficas

93

7. Anexos

CONSIDERAÇÕES GERAIS

RESUMO 
INTRODUÇÃo GERAL 


\section{INTRODUÇÃo GERAL}

A biodiversidade existente é resultado da dinâmica entre os processos naturais de especiação e extinção (Quental \& Marshall, 2013) e estima-se que 99\% de todas as espécies que já existiram estão hoje extintas (Novacek, 2001). A maior parte das espécies desapareceu ao longo dos tempos geológicos, pela extinção de fundo (Raup, 1986), mas houve cinco eventos catastróficos de extinção em massa, quando, pelo menos, três quartos das espécies desapareceram em um curto período de tempo geológico devido, entre outras causas, às mudanças globais no clima e na química atmosférica, pelo impacto de asteroides e vulcanismo (Jablonski, 1986; Bambach, 2006). Embora esses processos permaneçam importantes, as mudanças atuais na biodiversidade resultam direta ou indiretamente, quase exclusivamente, das atividades humanas, e estudos sugerem que um novo período de extinções em massa esteja em pleno andamento, 'Antropoceno' (May et al., 1995; Pimm et al., 1995; 2014; Barnosky et al., 2011)

Ainda que existam limitações reais na extensão e na qualidade de nosso conhecimento sobre os impactos das mudança antrópicas para a biodiversidade, a taxa de extinção de espécies estimada é de, pelo menos, três ordens de grandeza mais elevada do que a taxa de fundo e a grande maioria das populações de espécies ameaçadas continua a declinar (Millenium Ecosystem Assessment, 2005; Pimm et al., 2014; IUCN, 2015). As extinções causadas pelas atividades humanas não se restringem à atualidade. Há evidências da relação de hominínios modernos na extinção de espécies ao longo dos últimos 50.000 anos, porém as taxas de perda aceleraram acentuadamente no século passado com o aumento rápido da população humana (Malhi et al., 2014; Steffen et al., 2015).

Em um mundo cada vez mais modificado pelas atividades antrópicas, uma questão que preocupa é quanto da biodiversidade contemporânea sobreviverá em paisagens alteradas (Pereira, 2010). Biólogos conservacionistas estão preocupados com esta questão e tentam estimar as taxas e riscos atuais de extinções (IUCN, 2015), mas seu cálculo não é uma tarefa trivial, não apenas porque nosso conhecimento sobre a biodiversidade é limitado (Primack, 1993; Dirzo \& Raven, 2003; Pimm et al., 2014), mas, também, porque o intervalo de tempo entre a alteração no ambiente e o desaparecimento, de fato, das espécies 
pode ser bastante longo (Diamond, 1972).

0 atraso nos eventos de extinção vem sendo confirmado para muitas comunidades ecológicas (Kuusaaari et al., 2009; Brooks et al., 1999; Hanski, 2000; Hanski \& Ovaskainen, 2002; Lindborg \& Eriksson, 2004; Vellend et al., 2006; Dullinger et al., 2012). Esse atraso, ou débito de extinção, ocorre quando uma resposta ecológica a uma perturbação demora um tempo considerável para se tornar aparente (Tilman et al., 1994). 0 futuro da biodiversidade, portanto, vai depender de nossa capacidade de entender os efeitos dos impactos a longo prazo (Pauly, 1995; Metzger et al., 2009). Infelizmente, até para os táxons mais bem estudados e para regiões com grande diversidade de espécies, os monitoramentos que permitem quantificar as tendências da persistência da biodiversidade, após alterações nas condições iniciais, são ainda recentes (Ferraz et al., 2003; 2007; Stouffer et al., 2009).

Mesmo com essas dificuldades, alguns padrões relacionados à extinção de espécies já são evidentes. Sabe-se que entre as principais ameaças decorrentes diretamente das atividades humanas estão: a destruição e a degradação de habitats naturais (Nepstad et al., 1999; Fahrig, 2003; Laurance \& Peres, 2006) a introdução de espécies exóticas (Levine, 2000), a sobre-exploração de recursos biológicos (Pauly et al., 2002), os agentes patogênicos (Daszak et al., 2001), a poluição (Baillie et al., 2004) e as rápidas alterações climáticas (Parmesan et al., 1999; Thomas et al., 2004; Lewins et al., 2015). Embora os efeitos independentes de alguns destes distúrbios estejam cada vez mais bem caracterizados, o entendimento das interações entre padrões múltiplos de perturbações ainda permanece pouco claro (Laurance \& Useche, 2009; Turner, 2010). Sabe-se, entretanto, que as perturbações antrópicas raramente têm efeitos isolados na biodiversidade (Myers, 1995), mas são complexas, multifatoriais e frequentemente sinérgicas, podendo culminar em perdas irreversíveis para a biodiversidade (Terborgh et al., 2001; Laurance \& Peres, 2006; Laurance et al., 2011). Além dos efeitos das interações entre os diferentes distúrbios, o declínio de populações ou extinção de uma espécie têm efeitos interativos de amplo alcance no ecossistema para a sobrevivência de outras (Ewers \& Didham, 2006), podendo culminar em um efeito de extinções em cascata, com consequências desastrosas para a biodiversidade (Bascompte \& Jordano, 2007). 
De fato, muitos estudos sugerem que a resposta dos ecossistemas às alterações externas não é linear (Myers, 1995; Fahrig, 2001; 2003). Assim, influências cumulativas que, inicialmente parecem ter pouco efeito, podem levar a brusca e imprevisível alterações, uma vez que alguns limiares críticos são ultrapassados (Scheffer et al., 2001; Pardini et al., 2010). Além disso, a resistência e resiliência intrínseca às alterações podem variar com a simplificação antropogênica dos sistemas decorrentes das atividades humanas (Hughes 1994; Jackson, 1997; Pardini et al., 2010).

Dentre os distúrbios antrópicos, a perda e a fragmentação de habitat são atualmente as principais causas do declínio das espécies e populações (Fahrig, 2003). Essas alterações são decorrentes da necessidade de recursos que dependem de um novo uso da terra e conduzem a níveis elevados de conversão de habitat natural (FAO, 2010). Neste processo, 39\% da superfície terrestre disponível já foi convertida em uso agropecuário e assentamentos urbanos, e $37 \%$ dos remanescentes de habitat naturais encontram-se fragmentados, e imersos em matriz antrópica (Ellis et al., 2010). A redução de habitat disponível pela mudança da cobertura e uso da terra (Ewers \& Didham, 2006), ou seja, perda de habitat, é considerada a grande causa atual do declínio da biodiversidade (Fahrig, 2003; Millennium Ecosystem Assessment, 2005; Pimm et al., 2014).

Uma ameaça adicional decorrente da conversão de cobertura de complexos vegetacionais é a fragmentação do habitat, com graves consequências para muitas espécies (Saunders et al., 1991; Hanski, 2015). A fragmentação por mudanças antrópicas, tais como a supressão de vegetação natural para a construção de estradas ou para agricultura, leva habitats anteriormente contínuos a se tornarem subdivididos por uma matriz diferente da original (Fahrig, 2003; Ewers \& Didham, 2006; Laurance, 2008). A fragmentação de habitats pode resultar em fragmentos pequenos que, então, estão sujeitos a outras alterações associadas, tais como a redução da conectividade, o aumento do efeito de borda, a redução de qualidade por degradação ambiental, que agravam os efeitos da perda de habitat (Laurance et al., 2002; Wu et al., 2013).

Muito do conhecimento gerado sobre os efeitos da fragmentação de habitat na biodiversidade derivou da teoria de biogeografia de ilhas (MacArthur 
\& Wilson, 1967) que prevê que o tamanho da ilha e o seu isolamento, ou distância do continente determinam o número de espécies nas ilhas. Teoricamente, e em analogia à ilhas, fragmentos grandes e próximos à uma fonte (habitat contínuo) possuem maior número de espécies e fragmentos pequenos e isolados podem suportar apenas pequenas populações de espécies e tendem a ter menor número de espécies (MacArthur \& Wilson, 1967). Apesar da simplicidade e da importante contribuição desta teoria, há outros aspectos e escalas do efeito a serem considerados (Bender et al., 2003), principalmente no que diz respeito à quantidade de habitat disponível na paisagem (Pardini et al., 2010; Fahrig, 2013) e a persistência de populações interativas (Levins, 1970).

Para citar alguns autores, Andrén (1994) propôs que existe um limiar crítico na quantidade total de habitat remanescente na paisagem (20-30\%), abaixo do qual a conectividade é tipicamente rompida, e as espécies passam a ser mais dependentes, de fato, do tamanho e isolamento dos fragmentos. Pardini e colaboradores (2010) encontraram que a configuração do habitat seria importante em níveis intermediários de cobertura florestal na paisagem (30\%), que abaixo de um certo limiar haveria prejuízos para a resiliência ecológica, levando a mudanças irreversíveis na biodiversidade nestas paisagens fragmentadas. Fahrig (2013), contudo, propôs recentemente que a quantidade de habitat disponível na escala efetiva para as espécies é o que importa para a distribuição das espécies na paisagem, sendo desnecessário considerar a configuração do habitat. Hanski (2015) contesta essa hipótese, ressaltando a importância da configuração do habitat, além da quantidade de habitat, na determinação da persistência das espécies na paisagem. Villard \& Metzger (2014) argumentam que de fato, acima de uma quantidade de habitat, somente a perda de habitat importa na extinção local de espécies, mas que abaixo de certa quantidade de habitat, a configuração seria importante na mitigação dos efeitos de perda de habitat por meio da conectividade funcional, sendo que em níveis intermediários de proporção de cobertura haveria maior possibilidades de configuração e manejo das paisagens.

No contexto da paisagem, também importa a composição e a heterogeneidade dos habitats, e a capacidade da matriz de não habitat de facilitar o fluxo biológico entre os remanescentes (Taylor et al., 1993), 
juntamente com demais fatores relacionados à fragmentação (efeitos de borda) e perda de qualidade do habitat (Laurance \& Peres, 2006; Ewers \& Didham, 2006; Gardner et al., 2009).

Além do mais, espécies, populações e ecossistemas não estão apenas expostos a diferentes perturbações antrópicas, mas também diferem em sua vulnerabilidade e chances de persistirem a essas mudanças. Os riscos de extinção, portanto, geralmente variam biológica, taxonômica, mas também espacial e temporalmente (Bennett \& Owens 1997; Gaston \& Blackburn 1997; Owens \& Bennett, 2000; Purvis et al., 2000).

Quanto às características biológicas, espécies que desaparecem mais rapidamente de paisagens terrestres fragmentadas, muitas vezes têm grandes exigências de área e recursos, possuem baixa capacidade de dispersão e são especialistas de habitat primário, ou seja, têm alta sensibilidade às perturbações antrópicas (Tilman et al., 1994; Andrén et al., 1997; Laurence et al., 2001). Espécies com populações naturalmente instáveis também podem ser intrinsecamente mais vulneráveis à fragmentação, provavelmente porque suas populações flutuantes podem cair abaixo do limiar crítico para a sua persistência. Da mesma forma, espécies com baixas taxas de crescimento populacional podem ser menos propensas a se recuperar quando a população diminui muito e sofrem uma maior perda de diversidade genética (via deriva genética e endogamia) quando ocorrem gargalos populacionais (Saccheri et al., 1998). Alguns outros atributos biológicos podem ser associados à maior susceptibilidade das espécies às extinções e incluem: grande tamanho corpóreo, alto nível trófico, tempo de vida longo e baixa fecundidade, pequena abundância local (raridade) e/ou com distribuição geográfica restrita (Bennett \& Owens 1997; Purvis et al., 2000; Pimm et al., 2014). Sendo assim, diferentes táxons estão ameaçados por diferentes mecanismos, que interagem com diferentes características biológicas, resultando em um menor ou maior risco de extinção.

Também, o risco de extinção não é independente da filogenia, presumivelmente porque muitos dos traços biológicos associados com o maior risco de extinção tendem a ser compartilhados por espécies proximamente relacionadas (Gaston \& Blackburn, 1997; Bennet \& Owens, 1997). Existe também uma relação positiva entre a proporção de espécies em um táxon que 
são consideradas ameaçadas e a idade evolutiva desse táxon e a diversificação do grupo, o que sugere que as linhagens filogenéticas plesiomórficas e menos diversas são particularmente mais propensas à extinção (Gaston \& Blackburn 1997; Russel et al., 1998; Purvis et al., 2000).

Quanto à distribuição espacial, a maioria das extinções de espécies registradas desde 1500 ocorreu em ilhas (Baillie et al., 2004). A flora e a fauna insulares são especialmente vulneráveis à introdução antrópica de predadores, competidores e patógenos, enquanto que as espécies nos continentes não são tão sensíveis a essas modificações, provavelmente, por serem expostas a diferentes distúrbios, representando uma comunidade que é mais resistente à ameaças semelhantes (Balmford, 1996). No entanto, as previsões de extinções mais atuais indicam que as espécies nos continentes são as mais ameaçadas, especialmente, em florestas tropicais, em decorrência das altas taxas atuais de conversão de habitat, combinada com a alta diversidade e endemismo destas regiões (Myers et al., 2000; Pimm et al., 2014).

Temporalmente, dois padrões se destacam: a escala de perda está, em geral, aumentando desde de 1800 (Malhi et al., 2014), apesar das perdas de espécies causadas pelo homem pré-industrial terem sido substanciais (Jackson et al., 2001). 0 segundo padrão é que as ameaças antrópicas relacionadas à perda de espécies também mudam com o tempo; por exemplo, espécies invasoras e superexplotação (e.g. sobrecaça) foram as causas predominantes de extinções de aves em tempos históricos, enquanto a conversão de habitats, especialmente para a agropecuária, é a maior ameaça atual (Baillie et al., 2004) e há previsões de que as mudanças climáticas serão, no futuro próximo, uma grande ameaça (Thomas et al., 2004).

Cabe ressaltar que, embora muitas espécies desapareçam ou sofram declínio em suas populações em habitats fragmentados, outras podem aumentar drasticamente (Millennium Ecosystem Assessment, 2005). Espécies que são favorecidas por habitats de borda ou perturbados, que facilmente toleram matriz do entorno, ou cujos predadores ou competidores desaparecem com a fragmentação, muitas vezes se tornam mais abundantes e ocorrem em maior número de fragmentos (Laurance et al., 2001). Uma consequência dos padrões globais de extinção é a invasão de espécies e homogeneização biótica, que é o 
processo pelo qual comunidades bióticas tornam-se cada vez mais dominadas por um pequeno número de espécies generalistas, adaptadas às paisagens antropizadas (McKinney \& Lockwood, 1999).

A avaliação dos riscos de extinção em 2014, segundo a Lista Vermelha da União Internacional para a Conservação da Natureza e dos Recursos Naturais (IUCN, 2015) para 71.576 espécies (menos de 5\% das espécies atualmente descritas), principalmente terrestres e de água doce, mostrou que 21.286 espécies estão ameaçadas, sendo que 4.286 consideradas criticamente em perigo, e 860 espécies foram extintas desde 1500. A extinção de uma espécie representa o ponto final de uma série de extinções locais. Na verdade, populações podem ser extirpadas em um ritmo muito mais rápido do que o das espécies e as extinções locais podem ter sérias consequências para o funcionamento de ecossistemas locais (Hughes et al., 1997). Portanto, entender o processo de extinções locais é importante para a compreensão da relação entre a biodiversidade e as alterações nas paisagens, sendo que os declínios nas abundâncias de espécies podem ter implicações importantes para a funções ecossistêmicas, muito antes de qualquer espécie, de fato, ser extinta (Jackson et al., 2001).

Dentro do contexto sobre a extinção da biodiversidade em paisagens alteradas pelas atividades humanas, investigamos, principalmente, dois aspectos: i) a existência de atraso nas extinções locais de espécies após a perda e fragmentação de habitat e ii) os efeitos interativos entre a fragmentação e degradação de habitats na extinção de espécies. Poucos estudos investigaram o efeito do atraso nas extinções locais de espécies dentro do contexto amazônico (Wearn et al., 2012; Ferraz et al., 2003; 2007; Stouffer et al., 2009) e ainda há muito para ser entendido sobre os fatores de perturbação na qualidade do habitat local que levam à erosão de fragmentos, como fogo, extração seletiva de madeira, caça entre outros (Cochrane, 2003; Barlow \& Peres, 2004; Laurance \& Peres, 2006; Mestre et al., 2013). Assim, buscamos contribuir para o desenvolvimento teórico que embasa as práticas conservacionistas e o entendimento dos processos que levam à extinção da biodiversidade.

Avaliamos estes aspectos em uma paisagem real, hiperfragmentada inserida no Arco do Desmatamento Amazônico, que em termos absolutos é a 
mais agressiva fronteira de desmatamento no mundo (FAO, 2010). Esta região ao norte do Estado do Mato Grosso, na divisa com o Pará, era uma floresta contínua no final dos anos 70, contudo, foi rapidamente convertida em áreas destinadas à agropecuária. Hoje restam remanescentes de florestas em propriedades privadas, depauperados pela subsequente degradação por fogo, retirada de madeira e pela presença de gado e cercados principalmente de matriz de pastagem para gado.

A tese foi estruturada em dois capítulos, além da introdução, considerações finais e resumo geral. No primeiro capítulo, investigamos a existência de defasagens no tempo nas extinções em resposta a mudanças no tamanho e tempo desde o isolamento dos fragementos e então quantificamos as taxas de perda de espécies na paisagem estudada, examinando se esta variou entre os fragmentos de diferentes tamanhos. Inventariamos por pontos de escuta um grupo de aves conhecidamente sensíveis a perturbações antrópicas, os papaformigas, em 29 localidades amostrais e em dois períodos de amostragem (2004/06 e 2013). Baseamos nossa pesquisa em um modelo proposto por Gibson et al. (2013) derivado da teoria da biogeografia de ilhas, que inclui a área do fragmento e o tempo decorrido desde seu isolamento como variáveis preditoras da distribuição atual das espécies nos fragmentos, e também testamos para as espécies especialistas florestais. Qualitativamente, buscamos entender como ocorreu a alteração da composição das espécies na comunidade em relação às variáveis explanatórias, indicando as espécies mais vulneráveis à extinção, relacionando os padrões encontrados com a filogenia do grupo e comentando sobre algumas características das espécies.

No segundo capítulo, investigamos se houve efeitos de interações entre variáveis de degradação e fragmentação na distribuição das espécies de aves de sub-bosque na paisagem estudada. Para tal inventariamos as aves de sub-bosque por redes-de-neblina em 30 localidades amostrais. Testamos quais interações e quais variáveis de degradação de habitat estudadas (incidência de fogo, extração seletiva de madeira e presença de gado nos remanescentes florestais) e de fragmentação (área do fragmento, conectividade funcional e proporção de habitat em quatro escalas da paisagem) contribuíram para prever a distribuição da riqueza, abundância e composição de espécies de aves de sub-bosque nos 
fragmentos. Buscamos entender, qualitativamente, as mudanças na riqueza de espécies entre os fragmentos, investigando as alterações na composição de espécies na comunidade e sua relação com as variáveis estudadas. Também analisamos a diversidade beta da comunidade, buscando entender quanto da diversidade foi gerada por aninhamento e quanto por substituição, relacionando estes dois componentes às variáveis independentes estudadas. 
CONSIDERAÇõES GERAIS 


\section{CONSIDERAÇões GERAIS}

Os ecossistemas foram alterados da forma mais rápida e extensa no século passado, devido às intensas atividades humanas (Pereira, 2010; Malhi et al., 2014), sem precedentes na história da humanidade. Essa transformação no planeta ocorreu, em grande parte, para atender às demandas do rápido crescimento populacional, contribuiu para os ganhos líquidos substanciais no bem-estar humano e desenvolvimento econômico, mas suas consequências aos poucos estão se tornando aparentes (Millenium Ecosystem Assessment, 2005). Entre elas estão a destruição de habitats naturais e seus custos para as funções e serviços ecossistêmicos, o aumento do risco de mudanças bruscas, não lineares, o aumento da pobreza para alguns grupos de pessoas e a mudança e perdas da biodiversidade (Millenium Ecosystem Assessment, 2005).

Ainda que existam limitações em nosso conhecimento sobre os impactos das mudanças antrópicas para a biodiversidade, as sociedades modernas têm aumentado as taxas de extinção de espécies em pelo menos três vezes a do passado pré-histórico, o que sugere que estamos no sexto período de extinção em massa, denominado Antropoceno (May et al., 1995; Pimm et al., 1995; 2014; Malhi et al., 2014; Lewis et al., 2015). Além das espécies que já se extinguiram em decorrência das atividades humanas, um grande número de espécies em diversos grupos taxonômicos apresenta declínio, tanto do tamanho das populações como da distribuição geográfica ou ambos (IUCN, 2015). Acredita-se que a forma como a biodiversidade está sendo modificada pelas atividades humanas é, em grande extensão, irreversível (Nepstad et al., 1999; Lewis et al., 2015). A distribuição das espécies nas comunidades no mundo está cada vez mais homogênea, isto é, estão cada vez mais simplificadas e semelhantes entre si, principalmente como resultado da introdução de espécies invasoras (McKinney \& Lockwood, 1999).

A maior ameaça à integridade dos ecossistemas é a alteração no uso e cobertura da terra e a degradação dos habitats tropicais (Nepstad et al., 1999; Laurance \& Peres, 2006) que podem interagir de forma aditiva ou sinérgica para a extinção de espécies (Ewers \& Didham, 2006; Brook et al., 2008; Gardner et al., 2009). Por causa da crescente interação entre múltiplos distúrbios ambientais será cada vez mais difícil avaliar as implicações das mudanças nos ecossistemas 
(Myers, 1995; Laurance \& Peres, 2006; Turner, 2010). Outra dificuldade na avaliação nas taxas e riscos de extinções é decorrente da inércia nas respostas das comunidades biológicas às perturbações, e muitos efeitos demoram décadas ou muito mais tempo para se tornar aparentes (Tilman et al., 1994; Diamond, 1972).

Dentro deste contexto da crise da biodiversidade decorrente das atividades humanas, e das dificuldades que permeiam a avaliação dos riscos e taxas atuais de extinção, nossos resultados trazem importantes contribuições para o entendimento da extinção local de espécies em paisagens fragmentadas e degradadas. Embora existam alguns aspectos particulares das espécies e da paisagem estudadas, como a alta sensibilidade à alteração e relativa baixa capacidade de dispersão das espécies, também, o grande isolamento dos fragmentos e pouca permeabilidade da matriz de pastagem (baixa conectividade) e o fato da perda e fragmentação na região ter ocorrido recentemente ( $<40$ anos), há generalidade em nossas conclusões. Parte de nossos resultados advém do modelo proposto por Gibson et al. (2013) para explicar a extinção de pequenos mamíferos em ilhas verdadeiras em um reservatório hidroelétrico no sul da Tailândia e então confirmamos o poder explicativo do modelo em paisagens fragmentadas florestais. As demais interpretações do modelo são de cunho teórico e baseiam-se exclusivamente nos resultados do modelo. Também evidenciamos a importância de se considerar os efeitos das interações sinérgicas entre variáveis de perturbação para revelar as causas na perda de espécies e a contribuição da degradação ambiental nestas. Por fim, ressaltamos, a importância de se estudar o declínio de subpopulações e extinções de espécies localmente, uma vez que elas têm implicações diretas para a função dos ecossistemas, antes mesmo que a espécie seja globalmente extinta.

Os resultados do primeiro capítulo desta tese confirmam a existência de atraso na extinção local de espécies com a fragmentação e perda de habitat. 0 modelo proposto também permitiu calcular a taxa de extinção e de meia vida, ou seja, quanto tempo leva para que metade das espécies desapareça após o isolamento dos fragmentos. Obtivemos que, para o grupo estudado (papaformigas), mais da metade das espécies iniciais desapareceu em menos de duas décadas após o isolamento, independentemente do tamanho do fragmento. 
Entretanto, as taxas de extinção foram mais elevadas nos fragmentos grandes do que nos pequenos, pois estes perderam um maior número de espécies com o passar do tempo, enquanto que os fragmentos pequenos perderam muitas espécies imediatamente após o isolamento.

Neste capítulo foi possível elucidar que as espécies em fragmentos desaparecem em duas etapas: imediatamente após o isolamento, e com atraso. Além do mais, encontramos um limiar no tamanho dos fragmentos que determina a importância relativa na magnitude de perda de espécies nestas duas fases de extinção (imediata ou com atraso). Neste estudo, fragmentos menores de cerca de 150 hectares perderam grande parte de suas espécies imediatamente, enquanto que fragmentos maiores vão perder com o tempo a maioria de suas espécies comprometidas com a extinção. Sendo assim, o débito a ser pago nos fragmentos maiores é maior, assim como as taxas de extinções. Apesar das taxas de extinção elevadas nos fragmentos maiores isto não significa que fragmentos maiores irão perder as espécies mais rapidamente. Isso corrobora com a literatura (Kuussaari et al., 2009), que prevê que o atraso na extinção deverá ser mais prolongado quando o habitat modificado é ligeiramente diferente do necessário para assegurar a persistência da população a longo prazo (Brooks et al., 1999; Ferraz et al., 2003; 2007).

As taxas de extinção também variam ao longo do tempo, sendo maiores nos fragmentos logo após o isolamento, decaindo com o tempo desde o isolamento, novamente confirmando a literatura (Soulé et al., 1988; Hanski, 2000; Vellend et al., 2006). Tendo em vista o curto período em que mais da metade das espécies de papa-formigas desapareceu em paisagens fragmentadas e considerando que muitos fragmentos são menores do que o limiar aqui encontrado ( $<150$ ha), muitos dos fragmentos estudados já perderam a grande maioria de suas espécies de papa-formigas, o que chama a atenção para a curto janela de oportunidade disponível para mitigação dos impactos da fragmentação.

Nossos resultados também sugerem que as populações locais da maioria das espécies estão em declínio e que a família Grallariidae contém as espécies mais vulneráveis à extinção. As espécies Chamaeza nobilis, Formicarius analis, Grallaria varia e Myrmothera campanisona foram registradas em poucas localidades em 2004, na sua maioria em áreas controle e não foram registradas 
em 2013, embora isso não indique que as espécies desapareceram nestas florestas, mas é um indicativo de seu declínio com a fragmentação.

No segundo capítulo, verificamos a importância da incorporação das interações entre variáveis de fragmentação (tamanho do fragmento, conectividade funcional, proporção de habitat em diferentes escalas) e as relacionadas à degradação do habitat (incidência de fogo, extração seletiva de madeira, intrusão de gado) para explicar a riqueza, a abundância e a composição de espécies de aves de sub-bosque e do subgrupo das espécies especialistas florestais. A importância da incidência de fogo na distribuição de espécies amazônicas de aves de sub-bosque foi revelada apenas por causa de seu sinergismo com o tamanho do fragmento remanescente, sendo esta a principal causa da extinção de espécies nos fragmentos. Essa interação sinérgica entre a área do fragmento e a incidência de fogo também foi importante para explicar a variação no número de espécies florestais nos fragmentos, contudo este grupo também foi sensível aos efeitos aditivos da penetração do gado bovino nos remanescentes. A abundância e a composição de espécies nos fragmentos são influenciadas, principalmente, pelos efeitos combinados entre a área do fragmento e a presença de gado nos fragmentos. Também, encontramos que a alta diversidade beta dos fragmentos estudados é explicada pela substituição de espécies especialistas florestais por aves predominantemente encontradas em áreas perturbadas e do dossel de florestas, nos fragmentos com a presença de gado.

A variável área do fragmento esteve presente em todos os modelos, confirmando o reconhecido efeito da área de habitat na distribuição das espécies, abundâncias e composição nas comunidades, decorrente da disponibilidade de habitat. Porém, nossos resultados evidenciam que os efeitos da área florestal não foram suficientes por sí só para explicar os padrões observados, sendo que o fogo e a presença de gado têm grandes impactos sobre as aves de sub-bosque da região. Já a retirada de madeira teve pouca influência nestes padrões, sendo apenas importante para as abundâncias de espécies.

Nossa pesquisa reforça que, além de incluir variáveis que versam sobre a qualidade do habitat, é importante incluir as interações sinérgicas e aditivas entre variáveis explanatórias para revelar a importância velada de distúrbios 
ambientais, que de outra forma podem ficar mascarada, comprometendo a intepretação e os desdobramentos para a conservação da biodiversidade.

Concluímos assim, que políticas e ações conservacionistas devem considerar que os atrasos nas extinções locais de aves são muito curtos e principalmente imediatos nos fragmentos pequenos. Os efeitos interativos entre tamanho do fragmento e perda de qualidade pela subsequente degradação ambiental, como fogo e presença de gado, nos remanescentes florestais. Assim, reforçamos que a preservação dos remanescentes florestais não é suficiente para garantir a persistência das espécies, pois fragmentos pequenos estão mais sujeitos às queimadas e também são tipicamente mais influenciados pela presença de gado, além de perderem grande parte de suas espécies imediatamente após o isolamento. Embora a persistência de espécies em fragmentos grandes também seja influenciada por essas variáveis, suas espécies desaparecem gradativamente ao longo do tempo de relaxamento, podendo levar muitos anos até o débito de extinção ser completamente pago. Esses resultados corroboram a vulnerabilidade dos fragmentos pequenos e isolados na manutenção de espécies, que também sofrem mais com os efeitos de borda, invasão por espécies exóticas, entre outros (Laurance et al., 2002). Como uma boa estratégia de conservação recomendamos maior fiscalização para a coibição e controle de queimadas antrópicas, tão comuns nas fronteiras de desmatamento com o uso da técnica de corte-e-queima. Indicamos, também, a necessidade de utilização de cercas ao redor dos fragmentos para proteção contra a deterioração por entrada de gado, seja por incentivos ou por legislação. Como as taxas de extinções de espécies irão mudar no futuro vai depender de como, principalmente, a fragmentação e a degradação expandirão e quanto vamos conseguir proteger intacto para contrapor estas modificações. 
RESUMO 


\section{RESUMO}

As florestas tropicais contêm mais da metade de todas as espécies terrestres existentes, mas sofrem com a crescente influência das atividades humanas. A destruição e a degradação de habitats são, atualmente, as principais ameaças à biodiversidade. Embora exista uma extensa literatura sobre extinção de espécies em paisagens antropizadas, muitos aspectos ainda foram pouco estudados. Desta forma, buscamos contribuir para o entendimento sobre: i) o atraso e as taxas de extinções locais de espécies após a perda e a fragmentação do habitat florestal; e ii) como as interações entre variáveis de fragmentação e de degradação do habitat (perda de qualidade do habitat) podem agravar a taxa de extinção local de espécies. Para responder a estas questões, amostramos grupos de aves em uma paisagem intensamente fragmentada ao norte do Estado do Mato Grosso, no Arco do Desmatamento Amazônico. Para quantificar o débito de extinção, inventariamos as espécies de papa-formigas em dez pontos de escuta, durante três dias, em 29 localidades amostrais, durante dois períodos separados por quase uma década. Avaliamos o legado do histórico de fragmentação da paisagem na extinção local de espécies por meio de um modelo que considera o tamanho do fragmento e o tempo desde o seu isolamento. Para investigar o papel das interações entre a degradação e a fragmentação de habitats na extinção de espécies, consideramos a assembleia de aves de sub-bosque, inventariadas por redes-de-neblina durante 14.400 horas em 30 localidades amostrais. Utilizamos a seleção de modelos para determinar quais interações e variáveis de degradação e de fragmentação são melhores preditoras do número de espécies, abundância e a composição da avifauna nos fragmentos. Nossos resultados revelaram que há duas etapas para a extinção de espécies: a extinção imediata e a extinção com atraso. Mesmo considerando a extinção com atraso, mais da metade das espécies desaparece em menos de duas décadas nos fragmentos, independentemente do tamanho do fragmento. Encontramos que a grande maioria das espécies nos fragmentos pequenos ( $<150 \mathrm{ha}$ ) foi extinta localmente logo após o isolamento, enquanto que nos fragmentos grandes, a perda de espécies ocorre com o tempo e as taxas de extinção local são mais elevadas. Ademais, os efeitos das interações entre as variáveis de degradação e fragmentação de habitat contribuem para a extinção local de espécies na 
paisagem estudada. Identificamos que o sinergismo entre o tamanho do remanescente florestal e a incidência de fogo é a principal causa da extinção de espécies nos fragmentos. No entanto, a abundância e a composição de espécies na comunidade foram, principalmente, influenciadas pelas interações aditivas entre o tamanho do fragmento e a presença de gado. Observamos também que as alterações causadas pela presença do gado nos fragmentos resultam na substituição de espécies especialistas de habitat florestal e típicas de sub-bosque por espécies que habitam o dossel, bordas e áreas perturbadas. As implicações de nossos resultados para a conservação são: o intervalo de oportunidade para mitigação dos efeitos da fragmentação e perda de habitat, sobre os papaformigas, devido ao débito de extinção, mesmo que ainda presente, é muito curto para a implementação de ações eficazes para conservação, especialmente para os fragmentos pequenos, onde a perda da maioria das espécies ocorre imediatamente após o isolamento do fragmento. Portanto, ações de conservação deveriam otimizar seus esforços em fragmentos grandes (>150 ha), onde há maior chance de resguardar as espécies já comprometidas com a extinção. Além disso, as espécies nos fragmentos pequenos são negativamente e de forma mais intensa influenciadas pelos efeitos interativos com a incidência de fogo e penetração do gado. Concluímos que a preservação apenas dos remanescentes florestais não é suficiente para conservação da biodiversidade. Assim, as políticas públicas devem ser direcionadas à coibição de novos desmatamentos e queimadas, além do incentivo para a utilização de cercas ao redor dos remanescentes florestais em propriedades privadas.

Palavras chave: Amazônia, Comunidades de aves, Fragmentação florestal, Débito de Extinção, Degradação de habitat, Sinergismo ambiental, Conservação da biodiversidade 
AbStract 


\begin{abstract}
\end{abstract}
Tropical forests contain over half of all terrestrial species on Earth, but are succumbing to the growing impact of human activities. Habitat destruction and degradation are the main current threats to biodiversity. While there is an extensive literature on species extinction in human-modified landscapes, many aspects are yet to be explored. This study aims to contribute to our understanding of (i) the rates of time-lagged local extinctions of species in the aftermath of habitat loss and fragmentation; and (ii) the combined effects of habitat fragmentation and degradation (i.e. reduction in habitat quality) in aggravating rates of local extinctions. To develop this research, we sampled different functional groups of forest birds within an intensively fragmented landscape representative of the Arc of Deforestation of southern Brazilian Amazonia. To quantify the magnitude of extinction debt we inventoried antbirds at 29 forest sites during two periods, separated by nearly a decade. At each site, we carried out ten standardized point-counts over three days, which were validated with simultaneous digital recordings. We examined species extinction rates induced by historical landscape fragmentation using a model that considers forest fragment size and time since their isolation. In relation to interactions between habitat degradation and fragmentation, we considered all understorey birds inventoried by mist-nets during 14,400 hours at 30 sampling locations. We used model selection to determine which metrics and interactions of forest degradation (intensity of fires, selective logging and cattle presence within the forest remnants) and fragmentation (fragment size, amount of surrounding forest cover) best predicted the number, abundance and composition of species in the fragments. Our results revealed that there are two main stages for species extinctions: immediately after and time-lagged forest isolation. Even where there is a delay in species extinctions, over half of all species disappeared within less than two decades post-isolation, regardless of forest fragment size. We also found that the majority of species in small fragments $(<150$ ha) disappear immediately after isolation, whereas species losses in large fragments occur over time and they present higher local extinction rates. Moreover, interactions between habitat degradation and habitat fragmentation contributed to the local species extinctions in the studied landscape. Forest patch size operated 
synergistically with fire incidence as the main cause of local extinctions in fragments. However, the composition and abundance of species was influenced by the additive interactions between fragment size and cattle intrusion, which resulted in the replacement of understorey forest specialists with generalist species typically found in disturbed areas. The conservation implications of our results include: there is a narrow window of opportunity for mitigating the effects of habitat loss and fragmentation on antbirds, especially for small fragments, where most species were lost immediately after isolation. Conservation actions should be focused on large fragments ( $>150 \mathrm{ha}$ ) where there is greater potential for retaining species committed to extinction. Also, species in small fragments were more affected by the detrimental effects of fire incidence and cattle intrusion. We therefore conclude that the preservation of remaining forest fragments in itself is not enough for forest biodiversity conservation; public policy should be directed to fire suppression and fencing to deter cattle access to remaining forest fragments within private proprieties.

Keywords: Amazonia, Bird communities, Forest fragmentation, Extinction Debt, Habitat degradation, Environmental synergism, Biodiversity Conservation 


\section{REFERÊNCIAS BIBLIOGRÁFICAS GERAIS}




\section{REFERÊNCIAS BIBLIOGRÁFICAS GERAIS}

Achard, F., Eva, H. D., Stibig, H.-J., Mayaux, P., Gallego, J., Richards, T. and Malingreau, J.-P. 2002. Determination of deforestation rates of the world's humid tropical forests. Science 297, 999-1002.

Andrén, H. 1994. Effects of habitat fragmentation on birds and mammals in landscapes with different proportions of suitable habitat: a review. Oikos, 355366.

Andrén, H. 1997. Habitat fragmentation and changes in biodiversity. Ecological Bulletins, 171-181.

Baillie, J.E.M., Hilton-Taylor, C. and Stuart, S.N. (eds). 2004. 2004 IUCN Red List of Threatened Species. A Global Species Assessment. IUCN, Gland, Switzerland and Cambridge, UK.

Balmford, A. 1996. Extinction filters and current resilience: the significance of past selection pressures for conservation biology. Trends Ecol Evol 11, 193196.

Bambach, R. K. 2006. Phanerozoic biodiversity mass extinctions. Annu. Rev. Earth Planet. Sci. 34, 127-155.

Barlow, J. and Peres, C. A. 2004. Ecological responses to El Niño-induced surface fires in central Brazilian Amazonia: management implications for flammable tropical forests. Philosophical Transactions of the Royal Society of London B: Biological Sciences 359, 367-380.

Barnosky, A. D., Matzke, N., Tomiya, S., Wogan, G. O. U., Swartz, B., Quental, T. B., Marshall, C., McGuire, J. L., Lindsey, E. L. and Maguire, K. C. 2011. Has the Earth's sixth mass extinction already arrived? Nature 471, 51-57.

Bascompte, J. and Jordano, P. 2007. Plant-animal mutualistic networks: the architecture of biodiversity. Annual Review of Ecology, Evolution, and Systematics, 567-593.

Bender, D. J., Tischendorf, L. and Fahrig, L. 2003. Using patch isolation metrics to predict animal movement in binary landscapes. Landscape Ecol 18, 17-39.

Bennett, P. M. and Owens, I. P. F. 1997. Variation in extinction risk among birds: chance or evolutionary predisposition? Proceedings of the Royal Society of London B: Biological Sciences 264, 401-408.

Brook, B. W., Sodhi, N. S. and Bradshaw, C. J. A. 2008. Synergies among extinction drivers under global change. Trends Ecol Evol 23, 453-460.

Brooks, T. M., Pimm, S. L. and Oyugi, J. O. 1999. Time lag between deforestation 
and bird extinction in tropical forest fragments. Conserv Biol 13, 1140-1150.

Cochrane, M. A. 2003. Fire science for rainforests. Nature 421, 913-919.

Daszak, P., Cunningham, A. A. and Hyatt, A. D. 2001. Anthropogenic environmental change and the emergence of infectious diseases in wildlife. Acta tropica 78, 103-116.

Diamond, J. M. 1972. Biogeographic kinetics: estimation of relaxation times for avifaunas of southwest Pacific islands. Proceedings of the National Academy of Sciences 69, 3199-3203.

Dirzo, R. and Raven, P. H. 2003. Global state of biodiversity and loss. Annual Review of Environment and Resources 28, 137-167.

Dullinger, S., Gattringer, A., Thuiller, W., Moser, D., Zimmermann, N. E., Guisan, A., Willner, W., Plutzar, C., Leitner, M. and Mang, T. 2012. Extinction debt of highmountain plants under twenty-first-century climate change. Nat Clim Change $2,619-622$.

Ellis, E. C., Klein Goldewijk, K., Siebert, S., Lightman, D. and Ramankutty, N. 2010. Anthropogenic transformation of the biomes, 1700 to 2000. Global Ecol Biogeogr 19, 589-606.

Ewers, R. M. and Didham, R. K. 2006. Confounding factors in the detection of species responses to habitat fragmentation. Biological Reviews 81, 117-142.

Fahrig, L. 2001. How much habitat is enough? Biol Conserv 100, 65-74.

Fahrig, L. 2003. Effects of habitat fragmentation on biodiversity. Annual review of ecology, evolution, and systematics, 487-515.

Fahrig, L. 2013. Rethinking patch size and isolation effects: the habitat amount hypothesis. J Biogeogr 40, 1649-1663.

Ferraz, G., Russell, G. J., Stouffer, P. C., Bierregaard, R. O., Pimm, S. L. and Lovejoy, T. E. 2003. Rates of species loss from Amazonian forest fragments. Proceedings of the National Academy of Sciences 100, 14069-14073.

Ferraz, G., Nichols, J. D., Hines, J. E., Stouffer, P. C., Bierregaard, R. O. and Lovejoy, T. E. 2007. A large-scale deforestation experiment: Effects of patch area and isolation on Amazon birds. Science 315, 238-241.

Food and Agriculture Organization of the United Nations FAO. 2010. Global forest resources assessment 2010. FAO Forestry Pap., 147. FAO, Rome.

Gardner, T. A., Barlow, J., Chazdon, R., Ewers, R. M., Harvey, C. A., Peres, C. A. and Sodhi, N. S. 2009. Prospects for tropical forest biodiversity in a humanmodified world. Ecol Lett 12, 561-582. 
Gaston, K. J., Blackburn, T. M. and Lawton, J. H. 1997. Interspecific abundancerange size relationships: an appraisal of mechanisms. Journal of Animal Ecology, 579-601.

Gibson, L., Lynam, A. J., Bradshaw, C. J. A., He, F., Bickford, D. P., Woodruff, D. S., Bumrungsri, S. and Laurance, W. F. 2013. Near-complete extinction of native small mammal fauna 25 years after forest fragmentation. Science 341, 15081510 .

Hanski, I. 2000. Extinction debt and species credit in boreal forests: modelling the consequences of different approaches to biodiversity conservation. In Annales Zoologici Fennici 37, 271-280.

Hanski, I. and Ovaskainen, 0. 2002. Extinction debt at extinction threshold. Conserv Biol 16, 666-673.

Hanski, I. 2015. Habitat fragmentation and species richness. J Biogeogr 42, 989993.

Hughes, C. E. 1994. Risks of species introductions in tropical forestry. The Commonwealth Forestry Review, 243-252.

Hughes, B. B. 1997. Continuity and Change in World Politics: Competing Perspectives: Prentice Hall.

IUCN 2015. The IUCN Red List of Threatened Species. Version 2015.2. http://www.iucnredlist.org. último acesso em 20 de novembro de 2015.

Jablonski, D. 1986. Background and mass extinctions: the alternation of macroevolutionary regimes. Science 231, 129-133.

Jackson, J. B. C., Kirby, M. X., Berger, W. H., Bjorndal, K. A., Botsford, L. W., Bourque, B. J., Bradbury, R. H., Cooke, R., Erlandson, J. and Estes, J. A. 2001. Historical overfishing and the recent collapse of coastal ecosystems. Science 293, 629-637.

Kuussaari, M., Bommarco, R., Heikkinen, R. K., Helm, A., Krauss, J., Lindborg, R., Öckinger, E., Pärtel, M., Pino, J. and Roda, F. 2009. Extinction debt: a challenge for biodiversity conservation. Trends Ecol Evol 24, 564-571.

Laurance, W. F., Cochrane, M. A., Bergen, S., Fearnside, P. M., Delamônica, P., Barber, C., D'Angelo, S. and Fernandes, T. 2001. The future of the Brazilian Amazon. Science Washington 291, 438-439.

Laurance, W. F., Lovejoy, T. E., Vasconcelos, H. L., Bruna, E. M., Didham, R. K., Stouffer, P. C., Gascon, C., Bierregaard, R. O., Laurance, S. G. and Sampaio, E. 2002. Ecosystem decay of Amazonian forest fragments: a 22-year investigation. Conserv Biol 16, 605-618. 
Laurance, W. F. and Peres, C. A. 2006. Emerging threats to tropical forests: University of Chicago Press. Chicago

Laurance, W. F. 2008. Theory meets reality: how habitat fragmentation research has transcended island biogeographic theory. Biol Conserv 141, 1731-1744.

Laurance, W. F. and Useche, D. C. 2009. Environmental synergisms and extinctions of tropical species. Conserv Biol 23, 1427-1437.

Laurance, W. F., Camargo, J. L. C., Luizão, R. C. C., Laurance, S. G., Pimm, S. L., Bruna, E. M., Stouffer, P. C., Williamson, G. B., Benítez-Malvido, J. and Vasconcelos, H. L. 2011. The fate of Amazonian forest fragments: a 32-year investigation. Biol Conserv 144, 56-67.

Levine, J. M. 2000. Species diversity and biological invasions: relating local process to community pattern. Science $288,852-854$.

Lewis, S. L., Edwards, D. P. and Galbraith, D. 2015. Increasing human dominance of tropical forests. Science 349, 827-832.

Lindborg, R. and Eriksson, 0. 2004. Historical landscape connectivity affects present plant species diversity. Ecology 85, 1840-1845.

MacArthur, R. H. E. and 0. Wilson. 1967. The Theory of Island Biogeography. Monographs in Population Biology. Princeton University Press, Princeton, N.J.

Malhi, Y., Gardner, T. A., Goldsmith, G. R., Silman, M. R. and Zelazowski, P. 2014. Tropical forests in the Anthropocene. Annual Review of Environment and Resources 39, 125-159.

May, R. M.; Lawton, J. H.; Stork, N. E. 1995. in Extinction Rates. Oxford University Press. Oxford

McKinney, M. L. and Lockwood, J. L. 1999. Biotic homogenization: a few winners replacing many losers in the next mass extinction. Trends Ecol Evol 14, 450453.

Mestre, L. A. M., Cochrane, M. A. and Barlow, J. 2013. Long-term Changes in Bird Communities after Wildfires in the Central Brazilian Amazon. Biotropica 45, 480-488.

Metzger, J. P. 2009. Conservation issues in the Brazilian Atlantic forest. Biol Conserv 142, 1138-1140.

Millenium Ecosystem Assessment 2005. Ecosystems and human well-being: synthesis. Washington, DC: Island Press. Washington. 
Myers, N. 1995. Tropical deforestation: population, poverty and biodiversity in Swandon, T, ed., The economics and ecology of biodiversity decline: the forces driving global change, Cambridge University Press, 111-122.

Myers, N., Mittermeier, R. A., Mittermeier, C. G., Da Fonseca, G. A. B. and Kent, J. 2000. Biodiversity hotspots for conservation priorities. Nature 403, 853-858.

Nepstad, D., Soares-Filho, B. S., Merry, F., Lima, A., Moutinho, P., Carter, J., Bowman, M., Cattaneo, A., Rodrigues, H. and Schwartzman, S. 2009. The end of deforestation in the Brazilian Amazon. Science 326, 1350-1351.

Novacek, M. J. 2001. The biodiversity crisis: losing what counts: New Press. An American Museum of Natural History Book. The New Press. New York.

Owens, I. P. F. and Bennett, P. M. 2000. Ecological basis of extinction risk in birds: habitat loss versus human persecution and introduced predators. Proceedings of the National Academy of Sciences 97, 12144-12148.

Pardini, R., de Arruda Bueno, A., Gardner, T. A., Prado, P. I. and Metzger, J. P. 2010. Beyond the fragmentation threshold hypothesis: regime shifts in biodiversity across fragmented landscapes. Plos One 5, e13666.

Parmesan, C., Ryrholm, N., Stefanescu, C., Hill, J. K., Thomas, C. D., Descimon, H., Huntley, B., Kaila, L., Kullberg, J. and Tammaru, T. 1999. Poleward shifts in geographical ranges of butterfly species associated with regional warming. Nature 399, 579-583.

Pauly, D. 1995. Anecdotes and the shifting baseline syndrome of fisheries. Trends Ecol Evol 10, 430.

Pauly, D., Christensen, V., Guénette, S., Pitcher, T. J., Sumaila, U. R., Walters, C. J., Watson, R. and Zeller, D. 2002. Towards sustainability in world fisheries. Nature 418, 689-695.

Pereira, H. M., Leadley, P. W., Proença, V., Alkemade, R., Scharlemann, J. P. W., Fernandez-Manjarrés, J. F., Araújo, M. B., Balvanera, P., Biggs, R. and Cheung, W. W. L. 2010. Scenarios for global biodiversity in the 21st century. Science 330, 1496-1501.

Pimm, S. L., Russell, G. J., Gittleman, J. L. and Brooks, T. M. 1995. The future of biodiversity. Science 269, 347.

Pimm, S. L., Jenkins, C. N., Abell, R., Brooks, T. M., Gittleman, J. L., Joppa, L. N., Raven, P. H., Roberts, C. M. and Sexton, J. O. 2014. The biodiversity of species and their rates of extinction, distribution, and protection. Science 344, 1246752.

Primack, R. B. 1993. Essentials of conservation biology: Sinauer Associates Sunderland, Massachusetts. 
Purvis, A., Gittleman, J. L., Cowlishaw, G. and Mace, G. M. 2000. Predicting extinction risk in declining species. Proceedings of the Royal Society of London B: Biological Sciences 267, 1947-1952.

Quental, T. B. and Marshall, C. R. 2013. How the Red Queen drives terrestrial mammals to extinction. Science 341, 290-292.

Raup, D. M. 1986. Biological extinction in earth history. Science 231, 1528-1533.

Russell, G. J., Brooks, T. M., McKinney, M. M. and Anderson, C. G. 1998. Present and future taxonomic selectivity in bird and mammal extinctions. Conserv Biol $12,1365-1376$.

Rybicki, J. and Hanski, I. 2013. Species-area relationships and extinctions caused by habitat loss and fragmentation. Ecol Lett 16, 27-38.

Saccheri, I., Kuussaari, M., Kankare, M., Vikman, P., Fortelius, W. and Hanski, I. 1998. Inbreeding and extinction in a butterfly metapopulation. Nature 392, 491-494.

Saunders, D. A., Hobbs, R. J. and Margules, C. R. 1991. Biological consequences of ecosystem fragmentation: a review. Conserv Biol 5, 18-32.

Scheffer, M., Carpenter, S., Foley, J. A., Folke, C. and Walker, B. 2001. Catastrophic shifts in ecosystems. Nature 413, 591-596.

Steffen, W., Broadgate, W., Deutsch, L., Gaffney, O. and Ludwig, C. 2015. The trajectory of the Anthropocene: the great acceleration. The Anthropocene Review 2, 81-98.

Stouffer, P. C., Strong, C. and Naka, L. N. 2009. Twenty years of understorey bird extinctions from Amazonian rain forest fragments: consistent trends and landscape-mediated dynamics. Divers Distrib 15, 88-97.

Taylor, P. D., Fahrig, L., Henein, K. and Merriam, G. 1993. Connectivity is a vital element of landscape structure. Oikos, 571-573.

Terborgh, J., Lopez, L., Nunez, P., Rao, M., Shahabuddin, G., Orihuela, G., Riveros, M., Ascanio, R., Adler, G. H. and Lambert, T. D. 2001. Ecological meltdown in predator-free forest fragments. Science 294, 1923-1926.

Thomas, C. D., Cameron, A., Green, R. E., Bakkenes, M., Beaumont, L. J., Collingham, Y. C., Erasmus, B. F. N., De Siqueira, M. F., Grainger, A. and Hannah, L. 2004. Extinction risk from climate change. Nature 427, 145-148.

Tilman, D., May, R. M., Lehman, C. L. and Nowak, M. A. 1994. Habitat destruction and the extinction debt.. Nature 371, 65-66 
Turner, M. G. 2010. Disturbance and landscape dynamics in a changing world. Ecology 91, 2833-2849.

Vellend, M., Verheyen, K., Jacquemyn, H., Kolb, A., Van Calster, H., Peterken, G. and Hermy, M. 2006. Extinction debt of forest plants persists for more than a century following habitat fragmentation. Ecology 87, 542-548.

Villard, M. A. and Metzger, J. P. 2014. REVIEW: Beyond the fragmentation debate: a conceptual model to predict when habitat configuration really matters. Journal of Applied Ecology 51, 309-318.

Wearn, O. R., Reuman, D. C. and Ewers, R. M. 2012. Extinction debt and windows of conservation opportunity in the Brazilian Amazon. Science 337, 228-232.

Wu, J. 2013. Key concepts and research topics in landscape ecology revisited: 30 years after the Allerton Park workshop. Landscape Ecol 28, 1-11. 